\title{
FINITE ENERGY TRAVELLING WAVES FOR NONLINEAR DAMPED WAVE EQUATIONS
}

BY

\author{
EDUARD FEIREISL
}

Institute of Mathematics AVC̆R, Žitná 25, 11567 Praha 1, Czech Republic

1. Introduction. We show the existence of solitary waves resulting from a dynamic balance between dissipation and repulsive nonlinearity in a semilinear damped wave equation. More specifically, we examine the long-time behaviour of solutions to the Cauchy problem:

$$
\left\{\begin{array}{l}
u_{t t}+d u_{t}-\Delta u+m u=|u|^{\alpha-1} u \\
u=u(x, t), \quad x \in R^{N}, \quad N \geq 3, \quad t \in R^{+}, \\
u(x, 0)=u_{0}(x), \quad u_{t}(x, 0)=u_{1}(x), \quad x \in R^{N}
\end{array}\right.
$$

where $d, m$ are strictly positive constants and the exponent $\alpha$ satisfies

$$
1<\alpha<\alpha^{*} \quad \text { with } \quad \alpha^{*}=1+\min \left\{\frac{2}{N-2}, \frac{4}{N}\right\} .
$$

The basic quantity is the energy

$$
E\left(u, u_{t}\right) \equiv \frac{1}{2} \int_{R^{n}}|\nabla u|^{2}+m|u|^{2}+\left|u_{t}\right|^{2} d x-\frac{1}{\alpha+1} \int_{R^{n}}|u|^{\alpha+1} d x
$$

which represents a Lyapunov function of the problem, i.e., it is decreasing along any nonstationary trajectory of (1.1).

Employing the potential-well arguments of PAYNE-SATTINGER [16] one observes that any solution of (1.1) emanating from sufficiently small initial data exists globally for all $t \in R^{+}$and tends to zero with growing $t$. On the other hand, LEVINE [13, Part IV] showed that solutions corresponding to certain large data (with negative energy) blow up at a finite time.

In this paper, we shall deal with localized, finite energy states, i.e., with solutions to (1.1) that belong to neither class discussed above. A standard example of such a solution is, of course, a nonzero solution to the stationary problem:

$$
-\Delta w+m w=|w|^{\alpha-1} w, \quad w \in H^{1}\left(R^{N}\right) .
$$

According to KWONG [12], there is a positive, radially symmetric solution $w_{g}$ of $(1.4)$ unique (up to a spatial shift) in the class of positive solutions. On the other hand, the

Received February 1, 1995.

1991 Mathematics Subject Classification. Primary 35B40, 35L05. 
existence of an infinite sequence of solutions (changing sign) with energy going to infinity was proved by BERESTYCKI-LIONS [4]. All solutions of (1.4) except zero are unstable as solutions to the evolutionary problem (1.1) (see KELLER [11], SHATAH [19]).

It is well known that solutions of (1.1) satisfy the energy equality:

$$
\frac{d}{d t} E\left(u, u_{t}\right)(t)=-\int_{R^{N}}\left|u_{t}(t)\right|^{2} d x
$$

for all $t$ belonging to the existence interval $\left[0, T_{\max }\right)$. Consequently, for a global bounded solution the relation (1.5) may be integrated with respect to $t$ to deduce that $\left\|u_{t}\right\|_{L^{2}}$ must be "small" for large values of $t$. This justifies a conjecture that the long-time behaviour of bounded solutions of (1.1) should be somehow related to the stationary problem (1.4).

This is indeed the case as shown by the following result.

TheOREm 1.1. For any positive integer $k$ there exist initial data

$$
u_{0} \in H^{2}\left(R^{N}\right), \quad u_{1} \in H^{1}\left(R^{N}\right)
$$

such that the corresponding (strong) solution

$$
u \in \bigcap_{i=1}^{2} C^{i}\left(R^{+} ; H^{2-i}\left(R^{N}\right)\right)
$$

of the problem (1.1) is defined for all $t \in R^{+}$and

$$
\left\{\begin{array}{l}
\left\|u(\cdot, t)-\sum_{j=1}^{2 k}(-1)^{j} w_{g}\left(\cdot-x_{j}(t)\right)\right\|_{H^{1}} \rightarrow 0, \\
u_{t}(\cdot, t) \rightarrow 0 \text { in } L^{2}\left(R^{N}\right) \text { as } t \rightarrow \infty
\end{array}\right.
$$

where $w_{g}$ is the (unique) positive solution of (1.4) radially symmetric with respect to the origin and

$$
\left|x_{j}(t)\right| \rightarrow \infty, \quad \operatorname{dist}\left(x_{j}(t), x_{i}(t)\right) \rightarrow \infty \quad \text { for } i \neq j \text { as } t \rightarrow \infty .
$$

Thus the problem (1.1) possesses solutions that are global in time and split into a finite number of travelling waves that propagate in space with different velocities and locally "look" like the stationary solution $w_{g}$. As for different $k$ we find (different) solutions satisfying (1.6), the asymptotic behaviour of the dynamic system associated to (1.1) exhibits truly infinite-dimensional character in contrast with an analogous problem on a bounded spatial domain (cf. GHIDAGLIA-TEMAM [8]). Such solutions have all characteristic properties attributed to solitons as they are localized, finite energy states that are fundamentally nonlinear objects and so cannot be reached by perturbation theory from any linear state (REMOISSENET [18, Chapter 1]).

The number of waves appearing in (1.6) is even mainly because of the technique of the proof. On the other hand, it seems interesting to note that the existence of waves with an odd number of travelling components is not obvious. It is shown in [7] that if $N=1$ and $u$ is a solution of the problem (1.1) satisfying (1.6) with $k=1$, then $u$ converges to a fixed stationary solution.

The remaining part of the paper is devoted to the proof of Theorem 1.1. In Sec. 2, we review some standard facts concerning well-posedness of (1.1) and regularity properties of the solutions. 
In Sec. 3, we show that the asymptotic behaviour of bounded solutions may be described by a formula very similar to (1.6). The crucial observation is that any sequence of the form $\left\{u\left(\cdot, t_{n}\right)\right\}, t_{n} \rightarrow \infty$ where $u$ solves (1.1) represents a Palais-Smale sequence related to the stationary problem (1.4). Consequently, the technique of BENCI-CERAMI [2] and P. L. LIONS [14] can be used to obtain the desired result.

In Sec. 4, we construct solutions with the property

$$
0<c_{1} \leq\|u(\cdot, t)\|_{H^{1}} \leq c_{2} \quad \text { for all } t \in R^{+}
$$

(here and always the symbols $c_{i}, i=1,2, \ldots$ will denote positive constants). It is only at this stage of the proof where the specific shape of the nonlinearity plays a significant role. Specifically, we will see that boundedness of the energy of a trajectory $\left[u(t), u_{t}(t)\right]$, $t \in R^{+}$, yields boundedness in the energy space

$$
X \equiv H^{1}\left(R^{N}\right) \times L^{2}\left(R^{N}\right) .
$$

Note that such a trick is well known from the classical paper of AMBROSETTIRABINOWITZ [1] on the application of critical point theory to nonlinear elliptic problems.

Finally in Sec. 5, we take advantage of the invariance of the problem with respect to a certain group of reflections and construct solutions of (1.1) satisfying both (1.8) and

$$
\lim _{t \rightarrow \infty} E\left(u, u_{t}\right)(t)=2 k E\left(w_{g}, 0\right) \text {. }
$$

Such a solution will be shown to have all the properties claimed in the conclusion of Theorem 1.1. At this final step, the nonexistence result of ESTEBAN-LIONS [6] will play a crucial role in eliminating solutions that converge to a fixed stationary state.

2. Well-posedness and regularity of solutions. Let us begin with the linear problem:

$$
\left\{\begin{array}{l}
v_{t t}+d v_{t}-\Delta v+m v=g(x, t), \\
v(x, 0)=v_{0}(x), \quad v_{t}(x, 0)=v_{1}(x), \quad x \in R^{N} .
\end{array}\right.
$$

If $g=0$, it is well known (see, e.g., LIONS-MAGENES [15, Chapt. 3.8-3.9]) that the problem (2.1) is well posed and generates a $C_{0}$-semigroup $\left\{S_{t}\right\}$ on the Hilbert scale

$$
X_{\omega} \equiv H^{\omega}\left(R^{N}\right) \times H^{\omega-1}\left(R^{N}\right), \quad \omega \in[0,1] .
$$

Moreover, as $d>0$, there are constants $M, \delta>0$ such that

$$
\left\|S_{t}\right\|_{\mathcal{L}\left(X_{\omega}\right)} \leq M \exp (-\delta t) \quad \text { for all } t \in R^{+}, \omega \in[0,1]
$$

The solutions of the nonhomogeneous problem are given by the Duhamel formula

$$
\left[v(t), v_{t}(t)\right]=S_{t}\left[v_{0}, v_{1}\right]+\int_{0}^{t} S_{t-s}[0, g(s)] d s
$$

provided $g \in L_{\text {loc }}^{1}\left(R^{+}, H^{\omega-1}\left(R^{N}\right)\right)$.

By virtue of the hypothesis (1.2), the nonlinear term in Eq. (1.1) may be treated as a locally Lipschitz perturbation on the space $X$ and, consequently, the standard theory of evolution equations (see, e.g., PAZY [17, Chapt. 6]) yields the following result: 
Proposition 2.1. (i) For any data $\left[u_{0}, u_{1}\right] \in X$, there exists a function $u$ unique in the class

$$
u \in C\left(\left[0, T_{\max }\right), H^{1}\left(R^{N}\right)\right), \quad u_{t} \in C\left(\left[0, T_{\max }\right), L^{2}\left(R^{N}\right)\right)
$$

that solves the problem (1.1) in the sense of distributions on a maximal existence interval $\left[0, T_{\max }\right)$.

In case $T_{\max }<\infty$, we have

$$
\lim _{t \rightarrow T_{\max }} \|\left[u(t), u_{t}(t) \|_{X}=\infty .\right.
$$

(ii) For any $T<T_{\max }$ there is a neighbourhood $\mathcal{U}$ of $\left[u_{0}, u_{1}\right]$ in $X$ and a constant $\mathcal{L}(T, \mathcal{U})$ such that any solution $\left[v, v_{t}\right]$ of $(1.1)$ corresponding to the data

$$
\left[v(0), v_{t}(0)\right] \in \mathcal{U}
$$

is defined on $[0, T]$ and

$$
\|\left[v(T), v_{t}(T)\right]-\left[u(T), u_{t}(T)\left\|_{X} \leq \mathcal{L}\right\|\left[v(0), v_{t}(0)\right]-\left[u_{0}, u_{1}\right] \|_{X} .\right.
$$

The exponent $\alpha$ is subcritical in the sense that the superposition operator $u \rightarrow|u|^{\alpha-1} u$ may be differentiated both with respect to $x$ and $t$ to obtain a quantity bounded in $H^{\beta}\left(R^{N}\right)$ for a certain $\beta>-1$. Consequently, the standard bootstrap arguments (cf., e.g., GHIDAGLIA-TEMAM [9]) may be used to improve the regularity of the weak solutions.

Proposition 2.2. Assume that the initial data belong to the class

$$
u_{0} \in H^{2}\left(R^{N}\right), \quad u_{1} \in H^{1}\left(R^{N}\right) .
$$

Then the unique weak solution of problem (1.1) the existence of which is claimed in Proposition 2.1 is, in fact, a strong solution to the problem satisfying

$$
u \in \bigcap_{i=0}^{2} C^{i}\left(\left[0, T_{\max }\right), H^{2-i}\left(R^{N}\right)\right) .
$$

Note that, by virtue of (2.6), (2.7), all formulas derived in the future by means of multiplication of (1.1) by quantities containing various derivatives of $u$ may be justified, provided they make sense, even within the class of weak solutions by density arguments. In particular, the energy equality (1.5) holds for any weak solution of problem (1.1).

3. Asymptotic behaviour of bounded solutions. We start with a lemma on the asymptotic behaviour of Palais-Smale sequences that may be proved using the ideas of the concentrated compactness theory of P. L. LIONS [14, Theorem III.4]. The same result is also proved in BENCI-CERAMI [2, Lemma 3.1].

Proposition 3.1. Assume there is a sequence $\left\{u_{n}\right\} \subset H^{1}\left(R^{N}\right)$ such that

$$
\begin{gathered}
\left\|u_{n}\right\|_{H^{1}} \text { is bounded for } n \rightarrow \infty, \\
-\Delta u_{n}+m u_{n}-\left|u_{n}\right|^{\alpha-1} u_{n} \rightarrow 0 \text { in } H^{-1}\left(R^{N}\right) \text { as } n \rightarrow \infty .
\end{gathered}
$$


Then either $u_{n}$ converges to zero in $H^{1}\left(R^{N}\right)$ or there is a subsequence (not relabeled) and an integer $m \geq 1$ such that

$$
\left\|u_{n}-w_{1}-\sum_{j=2}^{m} w_{j}\left(\cdot-x_{n}^{j}\right)\right\|_{H^{1}} \rightarrow 0 \quad \text { as } n \rightarrow \infty
$$

where

$$
\left|x_{n}^{j}\right| \rightarrow \infty, \quad \operatorname{dist}\left(x_{n}^{i}, x_{n}^{j}\right) \rightarrow \infty \quad \text { for } i \neq j \text { as } n \rightarrow \infty
$$

and $w_{1}, w_{j} \neq 0, j=2, \ldots, m$, are (not necessarily distinct) solutions of problem (1.4).

Moreover, we have

$$
E\left(u_{n}, 0\right) \rightarrow \sum_{j=1}^{m} E\left(w_{j}, 0\right) \quad \text { as } n \rightarrow \infty .
$$

Our next goal is to show that the sequence $u_{n} \equiv u\left(t_{n}\right), t_{n} \rightarrow \infty$ where $u$ is a bounded solution of (1.1) satisfies the hypotheses of Proposition 3.1. To this end, assume that a global solution of (1.1) satisfies

$$
\left\|\left[u(t), u_{t}(t)\right]\right\|_{X} \leq c_{3} .
$$

Thus the energy $E\left(u, u_{t}\right)$ is bounded and we deduce from (1.5) that

$$
\int_{0}^{\infty} \int_{R^{N}} u_{t}^{2} d x d t \leq c_{4}
$$

Differentiating (1.1) with respect to $t$ and setting $v=u_{t}$ we obtain that $v$ solves the linear problem (2.1) with

$$
v(0)=u_{t}(0) \in L^{2}\left(R^{N}\right), \quad v_{t}(0)=u_{t t}(0) \in H^{-1}\left(R^{N}\right),
$$

and

$$
g=\alpha|u|^{\alpha-1} u_{t}
$$

By virtue of (1.2), (3.6), (3.7), we have

$$
g \in L^{\infty}\left(R^{+}, H^{-1}\left(R^{N}\right)\right) \cap L^{2}\left(R^{+}, H^{-1}\left(R^{N}\right)\right) .
$$

Now, we can use the linear theory on the space $L^{2} \times H^{-1}$, specifically the formulas (2.3), (2.4) together with the estimate (3.10) to conclude

$$
v(t)=u_{t}(t) \rightarrow 0 \text { in } L^{2}\left(R^{N}\right), \quad v_{t}(t)=u_{t t}(t) \rightarrow 0 \text { in } H^{-1}\left(R^{N}\right) \quad \text { as } t \rightarrow \infty .
$$

Consequently, setting $u_{n}=u\left(t_{n}\right)$ we get

$$
\begin{aligned}
-\Delta u_{n}+m u_{n}-\left|u_{n}\right|^{\alpha-1} u_{n}=-\left(u_{n}\right)_{t t}-d\left(u_{n}\right)_{t} & \rightarrow 0 \\
& \text { in } H^{-1}\left(R^{N}\right) \text { as } n \rightarrow \infty .
\end{aligned}
$$

Thus we are allowed to apply Proposition 3.1 to obtain:

Proposition 3.2. Assume there is a global solution $u$ of (1.1) satisfying (3.6).

Then for any sequence $t_{n} \rightarrow \infty$ either

$$
u\left(t_{n}\right) \rightarrow 0 \text { in } H^{1}\left(R^{N}\right), \quad u_{t}\left(t_{n}\right) \rightarrow 0 \text { in } L^{2}\left(R^{N}\right)
$$


or there is a subsequence (not relabeled) such that

$$
\begin{gathered}
\left\{\begin{array}{c}
\left\|u\left(t_{n}\right)-w_{1}-\sum_{j=2}^{m} w_{j}\left(\cdot-x_{n}^{j}\right)\right\|_{H^{1}} \rightarrow 0 \\
u_{t}\left(t_{n}\right) \rightarrow 0 \text { in } L^{2}\left(R^{N}\right) \text { as } t_{n} \rightarrow \infty
\end{array}\right. \\
E\left(u, u_{t}\right)\left(t_{n}\right) \rightarrow \sum_{j=1}^{m} E\left(w_{j}, 0\right)
\end{gathered}
$$

where the quantities $w_{j}, x_{n}^{j}, j=1, \ldots, m$, have the same properties as in Proposition 3.1 .

4. The existence of bounded solutions. In this section, we construct solutions satisfying (1.8). Let us start with two auxiliary results.

LEMma 4.1. Under the hypothesis (1.2), the zero solution is locally asymptotically stable, i.e., there is an $\varepsilon>0$ such that for all data satisfying

$$
\left\|\left[u_{0}, u_{1}\right]\right\|_{X}<\varepsilon
$$

the (unique) solution $u$ of (1.1) exists globally in time and

$$
u(t) \rightarrow 0 \text { in } H^{1}\left(R^{N}\right), \quad u_{t}(t) \rightarrow 0 \text { in } L^{2}\left(R^{N}\right) \quad \text { as } t \rightarrow \infty .
$$

Proof. Clearly, $u$ is a solution of the linear problem (2.1) with $g=|u|^{\alpha-1} u$. By virtue of the Sobolev embedding theorem we have

$$
\|g(t)\|_{L^{2}}^{2}=\int_{R^{N}}|u(t)|^{2 \alpha} d x \leq c_{5}\|u(t)\|_{H^{1}}^{2 \alpha} \text { for all } t \in\left[0, T_{\max }\right)
$$

where $\alpha>1$.

Consequently, if the data are sufficiently small, the relations (2.3), (2.4) imply exponential decay of the $X$-norm of the solution to zero. Q.E.D.

Lemma 4.2. Assume that a solution $u$ of (1.1) satisfies

$$
E\left(u, u_{t}\right)(t) \geq-c_{5} \text { for all } t \in\left[0, T_{\max }\right) .
$$

Then $T_{\max }=\infty$ and there is a constant $c_{6}$ such that

$$
\left\|\left[u(t), u_{t}(t)\right]\right\|_{X} \leq c_{6} \quad \text { for all } t \in R^{+} .
$$

Proof. Since the energy is bounded, the relation (1.5) yields

$$
\int_{0}^{T_{\max }} \int_{R^{N}} u_{t}^{2} d x d t \leq c_{7}
$$

Thus we can estimate the difference

$$
\begin{aligned}
\left\|u\left(t_{1}\right)-u\left(t_{2}\right)\right\|_{L^{2}} & \leq\left\|\int_{t_{1}}^{t_{2}} u_{t}(s) d s\right\|_{L^{2}} \\
& \leq \int_{t_{1}}^{t_{2}}\left\|u_{t}(s)\right\|_{L^{2}} d s \\
& \leq \sqrt{t_{2}-t_{1}} \sqrt{c_{7}} \text { for all } t_{1}<t_{2} \leq T_{\max }
\end{aligned}
$$

hence $\|u(t)\|_{L^{2}}$ is bounded on $\left[0, T_{\max }\right]$. 
On the other hand, integrating (1.5) with respect to $t$ we get

$$
\int_{R^{N}}|\nabla u(t)|^{2}+\left|u_{t}(t)\right|^{2}+m|u(t)|^{2} d x \leq c_{8}\left(1+\int_{R^{N}}|u(t)|^{\alpha+1} d x\right)
$$

where the last term may be estimated by means of the Sobolev interpolation inequality

$$
\int_{R^{N}}|v|^{\alpha+1} d x \leq c_{9}\left(\int_{R^{N}}|v|^{2} d x\right)^{\frac{2(\alpha+1)-N(\alpha-1)}{4}}\left(\int_{R^{N}}|\nabla v|^{2} d x\right)^{\frac{N(\alpha-1)}{4}} .
$$

By virtue of (1.2), we have always $\frac{N(\alpha-1)}{4}<1$ and, consequently, the relations (4.6), (4.7) yield

$$
\left\|\left[u(t), u_{t}(t)\right]\right\|_{X} \leq c_{10} \quad \text { on }\left[0, T_{\max }\right]
$$

In accordance with Proposition 2.1 (i), the solution $u$ is defined globally for all $t$ and (4.5) reads

$$
\int_{0}^{\infty} \int_{R^{N}} u_{t}^{2} d x d t \leq c_{7}
$$

To complete the proof, we have to show that the solution remains bounded in the energy norm. By virtue of $(4.7),(4.8)$, it suffices to prove that

$$
\|u(t)\|_{L^{2}} \leq c_{11} \quad \text { for all } t \in R^{+} .
$$

To this end, we multiply (1.1) by $u$ and integrate by parts to obtain

$$
\begin{aligned}
\int_{t_{1}}^{t_{2}} & \int_{R^{N}}|\nabla u|^{2}+m|u|^{2}-|u|^{\alpha+1} d x d t \\
= & \int_{R^{N}} u\left(t_{1}\right) u_{t}\left(t_{1}\right)-u\left(t_{2}\right) u_{t}\left(t_{2}\right) d x \\
& +\int_{t_{1}}^{t_{2}} \int_{R^{N}} u_{t}^{2} d x d t-d \int_{t_{1}}^{t_{2}} \int_{R^{N}} u u_{t} d x d t .
\end{aligned}
$$

Making use of (4.10) and the mean-value theorem we deduce that for an arbitrary time interval $I \subset R^{+}$of length 1 there are $t_{1}<t_{2}$ such that

$$
\left\{\begin{array}{l}
I \subset\left[t_{1}, t_{2}\right], \quad t_{2}-t_{1} \leq 3 \\
\left\|u_{t}\left(t_{1}\right)\right\|_{L^{2}},\left\|u_{t}\left(t_{2}\right)\right\|_{L^{2}} \leq \sqrt{c_{7}}
\end{array}\right.
$$

As the energy is nonincreasing, we have

$$
\int_{t_{1}}^{t_{2}} \int_{R^{N}} \frac{1}{2}\left(|\nabla u|^{2}+m\left|u^{2}\right|+\left|u_{t}\right|^{2}\right)-\frac{1}{\alpha+1}|u|^{\alpha+1} d x d t \leq\left(t_{2}-t_{1}\right) E\left(u_{0}, u_{1}\right) \leq c_{12} .
$$


Combining (4.10), (4.12), and (4.13) together with (4.14) we obtain

$$
\begin{aligned}
& \int_{t_{1}}^{t_{2}} \int_{R^{N}}|\nabla u|^{2}+|u|^{2}+\left|u_{t}\right|^{2} d x d t \\
& \quad \leq c_{13}\left(\left(\left\|u_{t}\left(t_{1}\right)\right\|_{L^{2}}+\left\|u_{t}\left(t_{2}\right)\right\|_{L^{2}}\right) \max _{t \in\left[t_{1}, t_{2}\right]}\|u(t)\|_{L^{2}}+1\right) \\
& \quad \leq c_{14}\left(1+\max _{t \in\left[t_{1}, t_{2}\right]}\|u(t)\|_{L^{2}}\right) .
\end{aligned}
$$

On the other hand, by means of $(4.6),(4.10)$ and the mean-value theorem we obtain

$$
\max _{t \in\left[t_{1}, t_{2}\right]}\|u(t)\|_{L^{2}} \leq c_{15}\left(1+\int_{t_{1}}^{t_{2}}\|u(s)\|_{L^{2}} d s\right) .
$$

Combining (4.15), (4.16) we get the desired estimate

$$
\int_{t_{1}}^{t_{2}} \int_{R^{N}}|\nabla u|^{2}+|u|^{2}+\left|u_{t}\right|^{2} d x \leq c_{16}
$$

for $t_{1}, t_{2}$ as in (4.13). Consequently, any time interval of length 3 contains a point $\xi$ such that

$$
\|u(\xi)\|_{L^{2}} \leq c_{17}
$$

which, together with (4.6), completes the proof of (4.11). Q.E.D.

Consider a couple of sets:

$$
\left\{\begin{aligned}
& \mathcal{C}_{0} \equiv \begin{array}{rl} 
& \left\{u_{0}, u_{1}\right] \in X \mid \text { the corresponding solution of }(1.1) \\
& \text { is defined for } t \in R^{+} \text {and } \\
& \left.\left[u(t), u_{t}(t)\right] \rightarrow 0 \text { in } X \text { as } t \rightarrow \infty\right\}
\end{array} \\
& \mathcal{C}_{-} \equiv\left\{\left[u_{0}, u_{1}\right] \in X \mid \text { the corresponding solution of }(1.1)\right. \\
&\text { satisfies } \left.\lim _{t \rightarrow T_{\max }} E\left(u, u_{t}\right)(t)<0\right\}
\end{aligned}\right.
$$

As a consequence of Proposition 2.1 (ii), Lemma 4.1, and the continuity of the energy, we get

LEMMA 4.3. Both sets $\mathcal{C}_{0}, \mathcal{C}_{-}$are nonvoid and open in $X$. Moreover,

$$
\mathcal{C}_{0} \cap \mathcal{C}_{-}=\varnothing
$$

By virtue of Lemma 4.2, we have

LEMMA 4.4. If

$$
\left[u_{0}, u_{1}\right] \in X \backslash \mathcal{C}_{-},
$$

then the solution exists for all $t \in R^{+}$and

$$
\|\left[u(t), u_{t}(t) \|_{X} \leq c_{18} \text { for all } t \in R^{+} .\right.
$$

Combining Lemma 4.3 and Lemma 4.4 along with a simple topological argument, namely that a connected set cannot be a union of two disjoint nonvoid open sets, we infer 
Proposition 4.5. Let $\chi:[0, q] \rightarrow X$ be a continuous curve such that $\chi(0) \in \mathcal{C}_{0}, \chi(q) \in$ $\mathcal{C}_{-}$.

Then there are initial data $\left[u_{0}, u_{1}\right]$ lying on $\chi$ such that the corresponding solution of (1.1) satisfies

$$
0<c_{19} \leq\left\|\left[u(t), u_{t}(t)\right]\right\|_{X} \leq c_{20} \quad \text { for all } t \in R^{+}
$$

5. The proof of Theorem 1.1. In this section, we complete the proof of Theorem 1.1. Let us start with a statement concerning the solutions of the stationary problem (1.4).

Proposition 5.1. There exists a unique positive, radially symmetric solution $w_{g}$ of $(1.4)$ attaining its maximum at the origin. The solution $w_{g}$ is a ground state, i.e., it minimizes the energy among all nonzero solutions of (1.4):

$$
0<E\left(w_{g}, 0\right)=\min \{E(w, 0) \mid w \neq 0 \text { solves }(1.4)\} .
$$

Moreover, there is a constant $\mu>0$ such that for any nonzero solution $w$ of (1.4) that is not a spatial shifts of $w_{g}$ or $-w_{g}$ we have

$$
E(w, 0)>E\left(w_{g}, 0\right)+\mu .
$$

Proof. The existence part is due to BERESTYCKI-LIONS [3]. They proved that the problem (1.4) possesses a nonzero, positive, radially symmetric and radially decreasing solution $w_{g}$ that minimizes the energy in the class of nonzero solutions (in fact, their result applies to a large class of nonlinear problems including (1.4)). KWONG [12] showed that a positive, radially symmetric solution of the problem (1.4) is unique up to a spatial shift. Consequently, all we have to prove is the relation (5.2).

Arguing by contradiction we assume that there is a sequence $\left\{w_{n}\right\}$ of solutions of $(1.4)$ that are not spatial shifts of $w_{g}$ or $-w_{g}$ such that

$$
E\left(w_{n}, 0\right) \rightarrow E\left(w_{g}, 0\right) .
$$

Multiplying Eq. (1.4) by $w_{n}$ and integrating by parts we obtain

$$
E\left(w_{n}, 0\right)=\left(\frac{1}{2}-\frac{1}{\alpha+1}\right) \int_{R^{N}}\left|\nabla w_{n}\right|^{2}+m\left|w_{n}\right|^{2} d x .
$$

By virtue of (5.3), (5.4), the sequence $\left\{w_{n}\right\}$ is bounded in $H^{1}\left(R^{N}\right)$ and, consequently, satisfies the hypotheses of Proposition 3.1. From (3.5), (5.3) we deduce that there is exactly one stationary solution, which we denote $\bar{w}$, appearing in (3.3). Consequently, passing to a subsequence and making a spatial shift if necessary, we may assume:

$$
w_{n} \rightarrow \bar{w} \text { in } H^{1}\left(R^{N}\right)
$$

where $\bar{w}$ is a solution of (1.4) satisfying

$$
E(\bar{w}, 0)=E\left(w_{g}, 0\right) .
$$

Using the standard bootstrap arguments and the $L^{p}$-estimates for elliptic problems we deduce that boundedness of the solutions $w_{n}, n=1,2, \ldots$ and $\bar{w}$ in $H^{1}\left(R^{N}\right)$ implies boundedness in the Sobolev space $W^{3, p}\left(R^{N}\right)$ with $p<\infty$ arbitrary. In particular, $\bar{w}$ 
is a classical solution of the problem minimizing the energy. According to COLEMANGLAZER-MARTIN [5], the function $\bar{w}$ is radially symmetric and radially monotone; hence, by KWONG [12], $\bar{w}$ is a spatial shift of $w_{g}$ or $-w_{g}$. Thus, using boundedness of $w_{n}$ in $W^{3, p}$, we infer

$$
w_{n} \rightarrow w_{g}\left(\text { or }-w_{g}\right) \text { in } H^{1}\left(R^{N}\right) \cap C^{2}\left(R^{N}\right) .
$$

In what follows, we shall assume convergence to $+w_{g}$, the other case being treated in a completely analogous way.

Since $w_{g}$ is strictly positive, we deduce from (5.7) the existence of $r>0$ such that

$$
w_{n}(x)>0 \text { for all }|x|=r \text { and all } n \text { large enough }
$$

and

$$
-\Delta w_{n}+b(x) w_{n}=0 \quad \text { for }|x|>r
$$

where the potential $b$ is bounded and strictly positive. By virtue of the maximum principle, we deduce from (5.8), (5.9) that

$$
w_{n}(x)>0 \text { for all } x,|x|>r \text { and all large } n .
$$

On the other hand, since $w_{g}$ is positive and the convergence in (5.7) is uniform on compact sets, we get

$$
w_{n}(x)>0 \text { for all } x,|x| \leq r .
$$

Consequently, since $w_{n}$ are positive everywhere, we can use the celebrated result of GIDAS-NI-NIRENBERG [10] to conclude that $w_{n}$ are radially symmetric at least for large $n$. But by the uniqueness result of KWONG [12], any radially symmetric positive solution of (1.4) is a spatial shift of $w_{g}$. Thus, all $w_{n}$ are spatial shifts of $w_{g}$ in contrast with our original assumption. Q.E.D.

Our strategy will be to prove Theorem 1.1 using Proposition 4.5. To this end, consider a system of unit vectors

$$
e_{j}=\left[\cos \left(\frac{\pi}{k} j\right), \sin \left(\frac{\pi}{k} j\right)\right], \quad j=1, \ldots, 2 k
$$

in $R^{2} \subset R^{N}$. Note that we write $\left[x_{1}, x_{2}\right]$ instead of $\left[x_{1}, x_{2}, 0, \ldots, 0\right]$ for simplicity. To this system, we associate a family of hyperplanes

$$
\mathcal{H}_{i}=\left\{x \in R^{N} \mid x \cdot h_{i}=0, i=1, \ldots, k\right\}
$$

where

$$
h_{i}=\left[-\sin \left(\frac{\pi}{2 k}(2 i-1)\right), \cos \left(\frac{\pi}{2 k}(2 i-1)\right], \quad i=1, \ldots, k .\right.
$$

Finally, let $U_{i}, i=1, \ldots, k$ denote the unitary transformation of reflection with respect to $\mathcal{H}_{i}$, i.e.,

$$
U_{i} x=x-2\left(x \cdot h_{i}\right) h_{i}, \quad x \in R^{N}, i=1, \ldots, k .
$$

Observe that

$$
U_{i}^{2}=\mathrm{Id}
$$


and

$$
U_{i}:\left\{e_{i}\right\}_{i=1}^{2 k} \rightarrow\left\{e_{i}\right\}_{i=1}^{2 k}
$$

is a one-to-one mapping such that

$$
\text { if } e_{l}=U_{i} e_{j} \text { for a certain } i \text {, then } l-j \text { is an odd integer. }
$$

Now, consider classes of symmetric functions:

$$
\mathcal{S}_{i}=\left\{v=v(x) \mid v\left(U_{i} x\right)=-v(x)\right\}, \quad i=1, \ldots, k .
$$

Observe that the $\mathcal{S}_{i}$ are invariant with respect to the solution semigroup of (1.1), i.e.,

$$
u_{0}, u_{1} \in \mathcal{S}_{i} \text { implies } u(t), u_{t}(t) \in \mathcal{S}_{i} \text { for all } t \in\left[0, T_{\max }\right)
$$

for any solution $u$ of (1.1). Indeed, the Laplacian commutes with both reflections and the inversion $v \approx-v$ and so does the nonlinearity (it is odd).

Consequently, the set $\mathcal{S}=\bigcap_{i=1}^{k} \mathcal{S}_{i}$ is invariant, i.e.,

$$
u_{0}, u_{1} \in \mathcal{S} \text { yields } u(t), u_{t}(t) \in S \text { for } t \in\left[0, T_{\max }\right) .
$$

Consider a function

$$
z(\lambda, x)=\sum_{j=1}^{2 k}(-1)^{j} w_{g}\left(x-\lambda e_{j}\right), \quad \lambda>0, x \in R^{N} .
$$

LEMma 5.2. We have $z(\lambda, \cdot) \in S$ for any fixed $\lambda>0$. Moreover, given $\varepsilon>0$ there is $\lambda_{0}$ such that

$$
E(z(\lambda, \cdot), 0) \leq 2 k E\left(w_{g}, 0\right)+\varepsilon
$$

for all $\lambda \geq \lambda_{0}$.

Proof. We have

$$
z\left(\lambda, U_{i} x\right)=\sum_{j=1}^{2 k}(-1)^{j} w_{g}\left(U_{i} x-\lambda e_{j}\right)
$$

where, since $w_{g}$ is radially symmetric,

$$
w_{g}\left(U_{i} x-\lambda e_{j}\right)=w_{g}\left(U_{i}\left(U_{i} x-\lambda e_{j}\right)\right)=w_{g}\left(x-\lambda U_{i} e_{j}\right) .
$$

By virtue of (5.14), (5.18), we get

$$
\begin{aligned}
z\left(\lambda, U_{i} x\right) & =\sum_{j=1}^{2 k}(-1)^{j} w_{g}\left(x-\lambda U_{i} e_{j}\right) \\
& =\sum_{l=1}^{2 k}(-1)^{j-l}(-1)^{l} w_{g}\left(x-\lambda e_{l}\right) \\
& =-z(\lambda, x), \quad \text { for all } i=1, \ldots, k .
\end{aligned}
$$

To observe (5.17), one has to realize that

$$
\operatorname{dist}\left(\lambda e_{j}, \lambda e_{i}\right) \rightarrow \infty, \quad i \neq j, \text { as } \lambda \rightarrow \infty
$$


and, consequently,

$$
\lim _{\lambda \rightarrow \infty} E(z(\lambda, \cdot), 0)=2 k E\left(w_{g}, 0\right) \text {. Q.E.D. }
$$

Keeping in mind the notation of Proposition 4.5, we consider a curve

$$
\left\{\begin{array}{l}
\chi:[0, q] \rightarrow X \\
\chi(s)=[s z(\lambda, \cdot), 0], s \in[0, q]
\end{array}\right.
$$

with parameters $\lambda, q$ to be determined below. Obviously,

$$
\chi(0)=[0,0] \in \mathcal{C}_{0} .
$$

Computing the energy we obtain

$$
E(\chi(s))=\frac{1}{2} s^{2} \int_{R^{N}}|\nabla z|^{2}+m|z|^{2} d x-\frac{s^{\alpha+1}}{\alpha+1} \int_{R^{N}}|z|^{\alpha+1} d x, \quad s \geq 0,
$$

and letting $\lambda \rightarrow \infty$ we deduce

$$
\left\{\begin{array}{l}
\int_{R^{N}}|\nabla z(\lambda, x)|^{2} d x \rightarrow 2 k \int_{R^{N}}\left|\nabla w_{g}(x)\right|^{2} d x \\
\int_{R^{N}}|z(\lambda, x)|^{2} d x \rightarrow 2 k \int_{R^{N}}\left|w_{g}(x)\right|^{2} d x \\
\int_{R^{N}}|z(\lambda, x)|^{\alpha+1} d x \rightarrow 2 k \int_{R^{N}}\left|w_{g}(x)\right|^{\alpha+1} d x .
\end{array}\right.
$$

On the other hand, $w_{g}$ is a solution of (1.4) and, consequently,

$$
0<Q \equiv \int_{R^{N}}\left|\nabla w_{g}\right|^{2}+m\left|w_{g}\right|^{2} d x \equiv \int_{R^{N}}\left|w_{g}\right|^{\alpha+1} d x .
$$

We deduce from (5.22)-(5.24) that the function

$$
s \rightarrow E(\chi(s))
$$

behaves for large $\lambda$ like

$$
s \rightarrow 2 k Q\left(\frac{s^{2}}{2}-\frac{s^{\alpha+1}}{\alpha+1}\right),
$$

i.e., attains its maximum at $s \approx 1$ and decays to $-\infty$ as $s \rightarrow \infty$. Thus given $\varepsilon>0$ arbitrary, we can fix the parameters $\lambda, q$ in such a way that

$$
\begin{gathered}
\chi(0)=[0,0], E(\chi(q))<0, \text { i.e., } \chi(q) \in \mathcal{C}_{-}, \\
\max _{s \geq 0} E(\chi(s)) \leq 2 k E\left(w_{g}, 0\right)+\varepsilon .
\end{gathered}
$$

Applying Proposition 4.5, we find data lying on $\chi$ such that the corresponding solution $u$ of the problem (1.1) is defined on $R^{+}$and satisfies

$$
0<c_{19} \leq\left\|\left[u(t), u_{t}(t)\right]\right\|_{X} \leq c_{20} \quad \text { for all } t \in R^{+} .
$$

Moreover, (5.25) yields

$$
\lim _{t \rightarrow \infty} E\left(u, u_{t}\right)(t) \leq 2 k E\left(w_{g}, 0\right)+\varepsilon
$$

and, by virtue of $(5.16)$,

$$
u(t), u_{t}(t) \in \mathcal{S} \quad \text { for all } t \in R^{+} .
$$

Our ultimate goal is to show that the solution just obtained has all the properties claimed in the conclusion of Theorem 1.1. 
Using Proposition 3.2 we obtain a sequence $t_{n} \rightarrow \infty$ such that $u\left(t_{n}\right), u_{t}\left(t_{n}\right)$ satisfy (3.14), (3.15). Our first claim is that the function $w_{1}$ in (3.14) must be zero. Clearly, we have $w_{1} \in \mathcal{S}$ since

$$
u\left(t_{n}\right) \rightarrow w_{1} \quad \text { in } H_{\mathrm{loc}}^{1}\left(R^{N}\right)
$$

and $u(t) \in \mathcal{S}$ for all $t$. In particular, $w_{1}$ is a solution of the problem (1.4) on the half-plane

$$
\Omega=\left\{x \mid x \cdot h_{i} \geq 0\right\}
$$

satisfying the boundary condition

$$
\left.\left.w_{1}\right|_{\partial \Omega} \equiv w_{1}\right|_{\mathcal{H}_{i}}=0 .
$$

It follows from the nonexistence results of ESTEBAN-LIONS [6] that $w_{1} \equiv 0$.

Next, using the relations $(3.15),(5.27)$ and the fact that $w_{g}$ minimizes the energy in the class of nonzero stationary solutions we deduce

$$
\left\{\begin{array}{l}
\left\|u\left(t_{n}\right)-\sum_{j=1}^{m} w_{j}\left(\cdot-x_{n}^{j}\right)\right\|_{H^{1}} \rightarrow 0 \\
u_{t}\left(t_{n}\right) \rightarrow 0 \text { in } L^{2}\left(R^{N}\right)
\end{array}\right.
$$

where

$$
\begin{aligned}
\left|x_{n}^{j}\right| \rightarrow \infty, \quad \operatorname{dist}\left(x_{n}^{i}, x_{n}^{j}\right) & \rightarrow \infty \quad \text { for } i \neq j \text { as } n \rightarrow \infty \\
m & \leq 2 k
\end{aligned}
$$

and $w_{j}$ are nonzero solutions of (1.4).

Now, our aim is to localize the points $x_{n}^{j}$. To this end, we introduce the sectors

$$
\begin{aligned}
\mathcal{V}_{j}=\left\{x=\left[x_{1}, x_{2}, \ldots, x_{N}\right]\right. & \in R^{N} \mid\left[x_{1}, x_{2}\right]=r[\cos \phi, \sin \phi], \\
r & \left.\geq 0, \phi \in\left[(2 j-1) \frac{\pi}{2 k},(2 j+1) \frac{\pi}{2 k}\right]\right\}, \quad j=1, \ldots, 2 k,
\end{aligned}
$$

i.e., $\mathcal{V}_{i}, \mathcal{V}_{i+k}, i=1, \ldots, k$ are the sectors determined by the hyperplanes $\mathcal{H}_{i}, \mathcal{H}_{i+1}$ (with the convention $\mathcal{H}_{k+1}=\mathcal{H}_{1}$ ) containing the vectors $e_{i}, e_{i+k}$ respectively. Observe that

$$
\begin{gathered}
R^{N}=\left\{\bigcup_{i=1}^{k} \mathcal{H}_{i}\right\} \cup\left\{\bigcup_{j=1}^{2 k} \operatorname{int} \mathcal{V}_{j}\right\}, \\
\operatorname{int} \mathcal{V}_{i} \cap \operatorname{int} \mathcal{V}_{j}=\varnothing \text { for } i \neq j .
\end{gathered}
$$

Lemma 5.3. There is a constant $C>0$ such that for any $l$ and any $n$ there is a $j$ such that

$$
\operatorname{dist}\left(x_{n}^{j}, \mathcal{V}_{l}\right) \leq C
$$

Proof. Assume the contrary, i.e., there is a sequence $n \rightarrow \infty$ and $l=l(n)$ such that

$$
\min _{j} \operatorname{dist}\left(x_{n}^{j}, \mathcal{V}_{l(n)}\right) \rightarrow \infty \quad \text { as } n \rightarrow \infty .
$$

Since $l(n)$ ranges only in a finite set, there is an $l$ which appears in (5.35) for infinitely many $n$. Passing to a subsequence, we may suppose

$$
\min _{j} \operatorname{dist}\left(x_{n}^{j}, \mathcal{V}_{l}\right) \rightarrow \infty \quad \text { as } n \rightarrow \infty
$$


On the other hand, since $u(t) \in \mathcal{S}$, we have

$$
\|u(t)\|_{H^{1}\left(\mathcal{V}_{i}\right)}=\|u(t)\|_{H^{1}\left(\mathcal{V}_{j}\right)} \quad \text { for all } i, j .
$$

Consequently, the relations $(5.29)$, (5.36) yield

$$
\left\|u\left(t_{n}\right)\right\|_{H^{1}\left(\mathcal{V}_{l}\right)} \rightarrow 0 \text { as } n \rightarrow \infty,
$$

which, by virtue of (5.37), implies

$$
u\left(t_{n}\right) \rightarrow 0 \quad \text { in } H^{1}\left(R^{N}\right),
$$

in contrast with (5.29). Q.E.D.

LEMMA 5.4. We have

$$
\min _{j, l} \operatorname{dist}\left(x_{n}^{j}, \partial \mathcal{V}_{l}\right) \rightarrow \infty \quad \text { as } n \rightarrow \infty .
$$

Proof. Assume the contrary, i.e., that there is a constant $K$ such that

$$
\operatorname{dist}\left(x_{n}^{j(n)}, \partial \mathcal{V}_{l(n)}\right) \leq K
$$

Similarly, as above, since there is only a finite number of indexes $j, l$, we can find a fixed couple $\bar{j}, \bar{l}$ such that

$$
\operatorname{dist}\left(x_{n}^{\bar{j}}, \partial \mathcal{V}_{\bar{l}}\right) \leq K \text { for all } n,
$$

in particular, there is an $\bar{i}$ such that

$$
\operatorname{dist}\left(x_{n}^{\bar{j}}, \mathcal{H}_{\bar{i}}\right) \leq K \quad \text { for } n \rightarrow \infty .
$$

Let $P$ be the orthogonal projection, $P: R^{N} \rightarrow \mathcal{H}_{\bar{i}}$. We define a sequence

$$
y_{n} \equiv P x_{n}^{\bar{j}} \text {. }
$$

By virtue of (5.30), (5.39), we get

$$
\begin{gathered}
x_{n}^{\bar{j}}-y_{n} \text { is convergent as } n \rightarrow \infty, \\
\left|x_{n}^{j}-y_{n}\right| \rightarrow \infty \text { for } j \neq \bar{j} \text { as } n \rightarrow \infty,
\end{gathered}
$$

passing to subsequences as the case may be.

Finally, we deduce from (5.29) that

$$
\left\|u\left(\cdot+y_{n}, t_{n}\right)-w_{\bar{j}}\left(\cdot-\left(x_{n}^{\bar{j}}-y_{n}\right)\right)-\sum_{j, j \neq \bar{j}} w_{j}\left(\cdot-\left(x_{n}^{j}-y_{n}\right)\right)\right\|_{H^{1}} \rightarrow 0 .
$$

By virtue of (5.40), we have

$$
w_{\bar{j}}\left(\cdot-\left(x_{n}^{\bar{j}}-y^{n}\right)\right) \rightarrow \bar{w} \text { in } H^{1}\left(R^{N}\right) \text { as } n \rightarrow \infty
$$

where $\bar{w}$ is a nonzero solution of (1.4).

On the other hand, if $U_{\bar{i}}$ is the reflection with respect to $\mathcal{H}_{\bar{i}}$, we have

$$
u\left(U_{\bar{i}} x+y_{n}, t_{n}\right)=u\left(U_{\bar{i}}\left(x+y_{n}\right), t_{n}\right)=-u\left(x+y^{n}, t_{n}\right)
$$

as $u \in \mathcal{S}$ and $U_{\bar{i}} P=P$. By virtue of (5.41), (5.42), we get

$$
u\left(\cdot+y_{n}, t_{n}\right) \rightarrow \bar{w} \text { in } H_{\mathrm{loc}}^{1}\left(R^{N}\right)
$$


which, together with (5.43), yields

$$
\bar{w} \equiv 0 \text { on } H_{\bar{i}} \text { in the sense of traces. }
$$

By the nonexistence result of ESTEBAN-LIONS [6] we get $\bar{w} \equiv 0$ on $R^{N}$-a contradiction. Q.E.D.

It follows from Lemma 5.3, Lemma 5.4 that for all $n$ sufficiently large, each set int $\mathcal{V}_{l}$ contains at least one point $x_{n}^{j}$, which together with (5.31), (5.33) implies

$$
m=2 k \text {. }
$$

By virtue of (3.15), (5.27), we have

$$
E\left(w_{j}, 0\right) \leq E\left(w_{g}, 0\right)+\varepsilon \text { for all } j=1, \ldots, 2 k .
$$

Thus by Proposition 5.1, the relation (5.45) yields that either

$$
w_{j}=w_{g} \quad \text { or } \quad w_{j}=-w_{g}
$$

or $w_{j}$ is a spatial shift of one of the above functions for any $j$. In any case, the asymptotic behaviour for $t_{n} \rightarrow \infty$ may be described by the formula

$$
\begin{gathered}
\left\|u\left(t_{n}\right)-\sum_{j=1}^{2 k}(-1)^{j} w_{g}\left(\cdot-x_{n}^{j}\right)\right\|_{H^{1}} \rightarrow 0 \text { as } t_{n} \rightarrow \infty, \\
u_{t}\left(t_{n}\right) \rightarrow 0 \text { in } L^{2}\left(R^{N}\right)
\end{gathered}
$$

with $x_{n}^{j}$ as in (5.30). Note that the factor $(-1)^{j}$ comes from the symmetry properties of $u$ and the fact that each sector $\mathcal{V}_{l}$ contains exactly one point $x_{n}^{j}$ for $n$ large.

Repeating the same procedure we see that the same holds for an arbitrary sequence $t_{n} \rightarrow \infty$, in particular, the number $2 k$ of "waves" in (5.46) is independent of $t_{n} \rightarrow \infty$ and we have always $w_{j}= \pm w_{g}$. Thus we can find the functions $x_{j}$ of the time $t$ satisfying (1.6), (1.7).

Theorem 1.1 has been proved.

6. Acknowledgment. The work was supported by Grant No. 201/94/1063 of the Grant Agency of the Czech Republic.

\section{REFERENCES}

[1] A. Ambrosetti and P. H. Rabinowitz, Dual variational methods in critical point theory and applications, J. Funct. Anal. 14, 349-381 (1973)

[2] V. Benci and G. Cerami, Positive solutions of some nonlinear elliptic problems in exterior domains. Arch. Rational Mech. Anal. 99 (4), 283-300 (1987)

[3] H. Berestycki and P. L. Lions, Nonlinear scalar field equations, I. Existence of a ground state, Arch. Rational Mech. Anal. 82, 313-346 (1983)

[4] H. Berestycki and P. L. Lions, Nonlinear scalar field equations, II. Existence of infinitely many solutions, Arch. Rational Mech. Anal. 82, 347-376 (1983)

[5] S. Coleman, V. Glazer, and A. Martin, Action minima among solutions to a class of Euclidean scalar field equations, Commun. Math. Phys. 58 (2), 211-221 (1978)

[6] M. J. Esteban and P. L. Lions, Existence and non-existence results for semilinear elliptic problems in unbounded domains, Proc. Royal Soc. Edinburgh 93 A, 1-14 (1982)

[7] E. Feireisl, Convergence to an equilibrium for semilinear wave equations on unbounded intervals, Dynamic Syst. Appl. 3 (3), 423-434 (1994) 
[8] J. M. Ghidaglia and R. Temam, Attractors for damped nonlinear hyperbolic equations, J. Math. Pures Appl. 66, 273-319 (1987)

[9] J. M. Ghidaglia and R. Temam, Regularity of the solutions of second order evolution equations and their attractors, Annali Scuola Normale Sup. Pisa Cl. Sci. 14, 485-511 (1987)

[10] B. Gidas, W. Ni, and L. Nirenberg, Symmetry and related properties by the maximum principle, Comm. Math. Phys. 68, 209-243 (1979)

[11] C. Keller, Stable and unstable manifolds for the nonlinear wave equation with dissipation, J. Differential Equations 50 (3), 330-347 (1983)

[12] M. K. Kwong, Uniqueness of positive solutions of $\Delta u-u+u^{p}=0$ in $R^{N}$, Arch. Rational Mech. Anal. 105, 243-266 (1989)

[13] H. A. Levine, Instability and nonexistence of global solutions of nonlinear wave equations of the form $P u_{t t}=-A u+\mathcal{F}(u)$, Trans. Amer. Math. Soc. 192, 1-21 (1974)

[14] P. L. Lions, On positive solutions of semilinear elliptic equations on unbounded domains, in Nonlinear Diffusion Equations and Their Equilibrium States, II, W. M. Ni, L. A. Peletier, J. Serrin, Editors, Math. Sci. Res. Inst. Publ. 13, Springer-Verlag, New York, Heidelberg, Berlin, 1988

[15] J. L. Lions and E. Magenes, Problèmes aux limites non homogènes et applications, I., Dunod, Paris, 1968

[16] L. E. Payne and D. H. Sattinger, Saddle points and instability of nonlinear hyperbolic equations, Israel J. Math. 22, 273-303 (1975)

[17] A. Pazy, Semigroups of linear operators and applications to partial differential equations, Appl. Math. Sci., vol. 44, Springer-Verlag, New York, Berlin, Heidelberg, Tokyo, 1983

[18] M. Remoissenet, Waves called solitons. Concepts and Experiments, Springer-Verlag, Berlin, Heidelberg, 1994

[19] J. Shatah, Unstable ground state of nonlinear Klein-Gordon equations, Trans. Amer. Math. Soc. 290 (2), 701-711 (1985) 